\title{
Novel Findings in Floating-Harbor Syndrome and a Mini-Review of the Literature
}

\author{
Pelin Ercoskun Cigdem Yuce-Kahraman \\ Department of Medical Genetics, Faculty of Medicine, Ataturk University, Erzurum, Turkey
}

\section{Established Facts}

- SRCAP is one of several proteins that act as coactivators for CREBBP which is associated with Rubinstein-Taybi syndrome. Nasolacrimal duct obstruction is defined in patients with Rubinstein-Taybi syndrome.

- Nasolacrimal duct obstruction, dystrophic toenails, and preauricular skin tag have not been previously reported in Floating-Harbor syndrome.

\section{Novel Insights}

- The fact that nasolacrimal duct obstruction has also been observed in Rubinstein-Taybi syndrome reinforces the idea that this finding is one of the features of the Floating-Harbor syndrome.

\section{Keywords}

Dystrophic toenails · Floating-Harbor syndrome .

Nasolacrimal duct obstruction · Rubinstein-Taybi syndrome - SRCAP gene

\section{Abstract \\ Floating-Harbor syndrome (FHS) is a rare autosomal domi- nant genetic disorder characterized by proportionate short stature with delayed bone maturation, lack of expressive lan- guage, and distinctive facial features including a large nose, long eyelashes, deeply set eyes, and triangular face. Muta- tions in the SRCAP gene cause truncated SNF2-related}

CREBBP activator protein (SRCAP) and lead to FHS. SRCAP is one of several proteins that act as coactivator for the CREBbinding protein which is associated with Rubinstein-Taybi syndrome (RSTS). This condition likely explains the phenotypic overlap between FHS and RSTS. Herein, we report on a patient with FHS who also had dystrophic toenails, preauricular skin tag, and nasolacrimal duct obstruction which is also defined in patients with RSTS. In summary, the fact that especially nasolacrimal duct obstruction has also been observed in RSTS reinforces the idea that this finding is one of the features of FHS. Assessment of the lacrimal system and examination of skin and nails should be suggested in patients with FHS.
Pelin Ercoskun

Department of Medical Genetics, Faculty of Medicine Ataturk University, Yakutiye

Erzurum 25240 (Turkey)

pelin.ercoskun@atauni.edu.tr 


\section{Introduction}

Floating-Harbor syndrome (FHS) (OMIM \#136140) is a very rare genetic disorder defined by short stature, delayed bone mineralization, speech impairment, and dysmorphic facial features. The name of the disorder comes from the 2 hospitals (Boston Floating Hospital and Harbor General Hospital) from where the first 2 cases were reported [Pelletier and Feingold, 1973; Leisti et al., 1975]. To date, more than 60 cases have been reported in the medical literature [Nikkel et al., 2013].

The SRCAP gene, located in chromosome 16p11.2, is the causative gene of FHS. This gene encodes the SNF2-related activator protein (SRCAP) which plays an essential role in chromatin remodeling and gene expression and acts as a coactivator for CREB-binding protein (CREBBP) that is responsible for RubinsteinTaybi syndrome (RSTS) [Hood et al., 2012]. The association between SRCAP and CREBBP genes likely explains the clinical overlap between FHS and RSTS. Although most cases of FHS are sporadic, genomic analyses show that the inheritance of this syndrome is autosomal dominant, as suggested by reported cases of parent-to-child transmission [Arpin et al., 2012; Messina et al., 2016].

The diagnosis of this disorder can be difficult without the distinctive facial findings like triangular face with a broad bulbous nose, deeply set eyes, long eyelashes, thin lips, wide columella, short philtrum, and relatively wide mouth. Short stature with delayed bone age and finger anomalies like brachydactyly, broad thumbs, and clinodactyly are important findings for the diagnosis. Additionally, congenital heart defects, urogenital anomalies, seizures, gastroesophageal reflux, constipation, recurrent otitis media, cleft lip/palate, and dental anomalies have also been reported in the literature [White et al., 2010; Nikkel et al., 2013].

In this paper, we report on a patient with FHS who had a preauricular skin tag, dystrophic toenails, and nasolacrimal duct obstruction which is also defined in patients with RSTS.

\section{Case Report}

The patient, a 2.5-year-old male, is the third child of a healthy nonconsanguineous Turkish couple. Following an uncomplicated pregnancy, he was born on time via vaginal delivery with a birth weight of $3,055 \mathrm{~g}$ (10th-25th percentile) and a length of $50 \mathrm{~cm}$ (50th percentile). The

New Findings in Floating-Harbor Syndrome family history was unremarkable. On the first day of his life, he developed a high body temperature and was hospitalized in a pediatric care unit due to suspected neonatal sepsis and treated with antibiotics.

Later, when he was 2.5 years old, he was referred to our clinic because of his dysmorphic facial features and speech impairment. On clinical examination, he had a triangular face with prominent nose, long eyelashes, deeply set eyes, prominent ears, a preauricular skin tag, short philtrum, and thin upper lip. Small teeth with abnormal shape and tooth cavities were seen in oral evaluation. Hirsutism, especially in the facial area, and dystrophic toenails were noted. He had clinodactyly of 5th fingers and broad fingertips (Fig. 1). He was $74 \mathrm{~cm}$ tall ( $<3 \mathrm{rd}$ percentile) and weighed $7 \mathrm{~kg}(<3 \mathrm{rd}$ percentile). Occipitofrontal circumference was $47.5 \mathrm{~cm}$ (15th percentile). Due to short stature, hand-wrist radiography was performed, revealing delayed bone maturation (compatible with 1 year old). Frequently he had otitis media infection and pneumonia which required hospitalization. A CT scan of the temporal bones showed fluid densities in the bilateral middle ear cavity and mastoid air cells, suggestive of otomastoiditis developing on the basis of chronic otitis media. Hypospadias, bilateral reducible inguinal hernia and retractile testicles were also noted. His urinary ultrasound imaging was normal, and scrotal ultrasound, performed at infant period, detected that testicles were in the inguinal region. Additionally, he had a submucous cleft palate (non-operated) and difficulties in feeding. Atrial septal defect (secundum type) and patent ductus arteriosus were seen on his echocardiogram performed in the neonatal period. He had various symptoms of the gastrointestinal system like gastroesophageal reflux and constipation.

He had purulent discharge from the eyes due to the infection of his lacrimal system. Bilateral nasolacrimal duct obstruction was noted on his ophthalmologic assessment.

\section{Results}

Results of karyotype analysis and FISH analysis of the 22q.11 locus were normal. Following the clinical diagnosis of FHS, molecular genetic analysis of the SRCAP gene was carried out. Molecular sequencing of the SRCAP gene revealed a heterozygous truncating mutation in exon 34, (NM_006662.3):c.7330C>T;p.Arg2444*. The clinical diagnosis was confirmed by the result of this genetic testing. The variant was not seen in the patient's parents. 
Fig. 1. The findings of our patient with Floating-Harbor syndrome. a Triangular face with prominent nose, long eyelashes, deeply set eyes, prominent ears, short philtrum, and hirsutism in the facial area. $\mathbf{b} \mathrm{Pu}$ rulent discharge from the eyes due to the infection of his lacrimal system. c Preauricular skin tag. d Dystrophic toenail. e Clinodactyly of 5 th finger and broad fingertips.
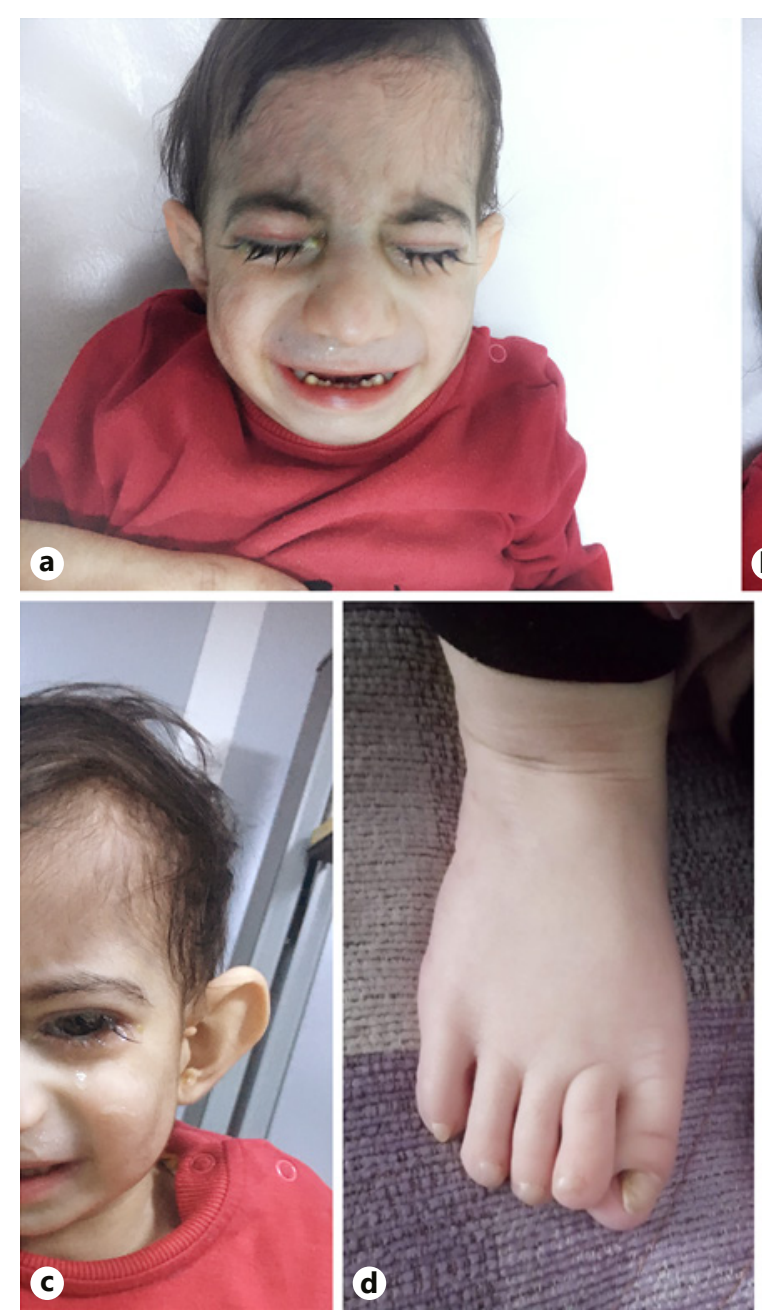
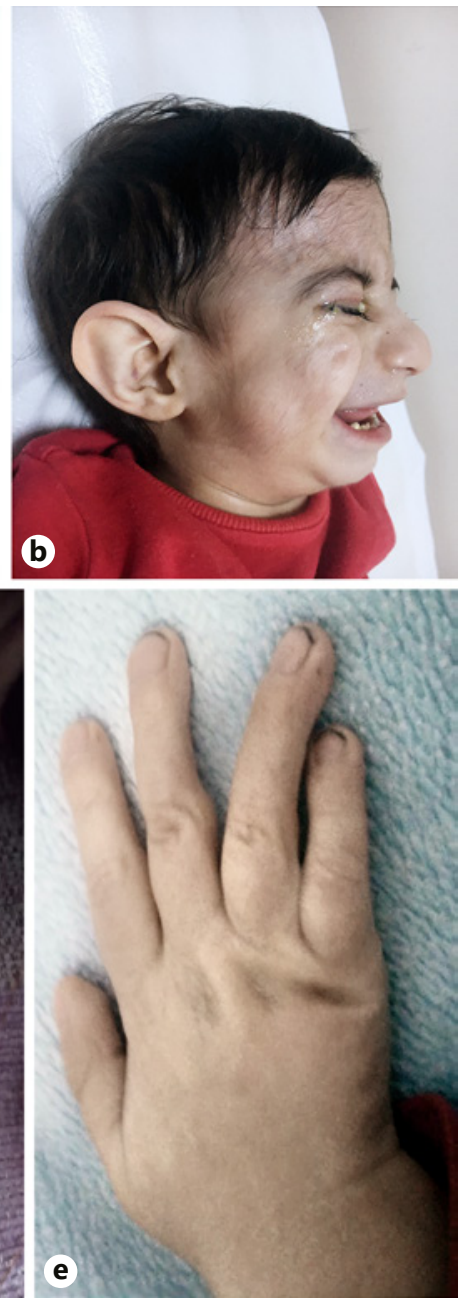

\section{Discussion}

In this paper, we report a detailed clinical evaluation and genetic analysis of a patient with FHS. The patient had nasolacrimal duct obstruction, dystrophic toenails, and preauricular skin tag in addition to classical findings of FHS.

FHS was first described in 1973 [Pelletier and Feingold, 1973] and then in 1975 [Leisti et al., 1975] as a genetic disorder defined by short stature, lack of speech, and dysmorphic facial gestalts. The syndrome is caused by heterozygous mutations in the SRCAP gene which encodes the SNF-2 related chromatin remodeling ATPase. This protein is one of several proteins that serve as coactivators for CREB-binding protein, known as CBP, whose mutations cause RSTS [Hood et al., 2012]. Mutations in the SRCAP gene were predicted to result in the formation of C-terminally truncated SRCAP. This protein variant may disrupt the binding of the wild type SRCAP to the DNA and its chromatin targets, known as dominant negative effect. With these mutations of the SRCAP gene, the expression levels of the genes which are responsible for the onset of differentiation and developmental processes of the embryo are affected [Messina et al., 2016].

In their study, Hood et al. [2012] noted that all heterozygous variants were truncating (nonsense or frameshift), and all of them were clustered in exon 34. Additionally, Nikkel et al. [2013] reported that all of the mutations in the SRCAP gene were truncating, and the most frequent mutation was p.Arg2444*; c.7330C>T which was also detected in our case. Although the majority of the mutations reported in the literature are clustered in hot spots of exon 34, the final exon of the SRCAP gene, some studies have 
shown that SRCAP gene mutations are not restricted to exon 34 [Kehrer et al., 2014; Seifert et al., 2014].

Clinical manifestations of FHS include the triad of short stature with delayed bone maturation, delayed impressive language, and dysmorphic facial features. Despite of changing with age, facial features, particularly the nose, are the most important findings for the diagnosis. In infancy, the facial findings are subtle. Patients have a triangular facial shape with a nose which is narrow at the root and broadens to the tip, long eyelashes, a relatively wide mouth, and deep-set eyes. Thin upper vermillion, short philtrum, and low hanging columella are also among the findings of the disease. The nose becomes more conspicuous with age [White et al., 2010; Nikkel et al., 2013].

Proportionate short stature is one of the cardinal features of the disease. In the prepubertal period, delayed bone age is observed, and it is expected to normalize with the pubertal period. Although the majority of patients have growth hormone and IGF-1 values within the normal range, patients with growth hormone deficiency have also been reported. Some patients benefit from recombinant human growth hormone replacement therapy, but there is not yet a consensus in the literature about the effects of this treatment on adult height. Additionally, patients with precocious puberty have rarely been defined [Garcia et al., 2012; Homma et al., 2019].

Delay of expressive language is seen with varying severity in patients with FHS. Mild intellectual disability may be observed in some patients. Anxiety, attention deficit hyperactivity disorder, and rigid mannerism have been described as behavioral phenotype in these individuals [Nikkel et al., 2013].

A varying range of different clinical findings such as recurrent otitis media, hearing loss, strabismus, dental anomalies, cleft lip and palate, cardiac malformation, gastrointestinal motility abnormalities like reflux or constipation, celiac disease, genitourinary anomalies like cryptorchidism, renal collecting system anomalies or renal calculi have been reported in different studies [Nikkel et al., 2013; Shields et al., 2019; Ko et al., 2020].

Congenital nasolacrimal duct obstruction is a frequent anomaly in pediatric patients with a prevalence that ranges from 5 to $20 \%$. This anomaly leads to a lack of the nasolacrimal drainage system, and the patient presents with overflow of tears, known as epiphora, caused by the persistence of a membrane in the distal segment of the nasolacrimal duct. Although nasolacrimal duct obstruction is associated with various syndromes such as Down syndrome, RSTS or Treacher Collins syndrome, to the best of our knowledge, it has not been previously reported in patients with FHS. RSTS is an autosomal dominant disorder marked by striking facial features, broad and angulated thumbs and halluces, short stature, and intellectual disability. CREBBP is the causative gene in RSTS and SRCAP acts as coactivator protein for this gene. The association between these 2 proteins explains the clinical overlap between FHS and RSTS.

In summary, the fact that nasolacrimal duct obstruction has also been found in RSTS, reinforces the idea that this finding is one of the features of FHS. Additionally, dystrophic toenails and preauricular skin tag have not been noted in patients with FHS yet. In conclusion, assessment of the lacrimal system and examination of skin and nails should be suggested in patients with FHS [van Genderen et al., 2000; Hutchinson and Sullivan, 2015; Ali and Paulsen, 2017; Vagge et al., 2018].

\section{Acknowledgement}

We would like to thank the patient and his parents for their cooperation.

\section{Statement of Ethics}

This study was performed in accordance with the Declaration of Helsinki principles. Written informed consent was obtained from the family.

\section{Conflict of Interest Statement}

The authors have no conflicts of interest to declare.

\section{Funding Sources}

The authors confirm independence from sponsors; the content of the article has not been influenced by sponsors.

\section{Author Contributions}

P.E. was involved in the collection of clinical data, clinical examination and wrote the manuscript. C.Y.K. conducted clinical evaluations and managed the patient. 


\section{References}

Ali MJ, Paulsen F. Syndromic and Nonsyndromic Systemic Associations of Congenital Lacrimal Drainage Anomalies: A Major Review. Ophthal Plast Reconstr Surg. 2017;33(6):399-407.

Arpin S, Afenjar A, Dubern B, Toutain A, Cabrol $S$, Héron D. Floating-Harbor Syndrome: Report on a Case in a Mother and Daughter, Further Evidence of Autosomal Dominant Inheritance. Clin Dysmorphol. 2012;21(1): 11-4.

García RJ, Kant SG, Wit JM, Mericq V. Clinical and genetic characteristics and effects of longterm growth hormone therapy in a girl with Floating-Harbor syndrome. J Pediatr Endocrinol Metab. 2012;25(1-2):207-12.

Homma TK, Freire BL, Honjo R, Dauber A, Funari MFA, Lerario AM, et al. Growth and Clinical Characteristics of Children With Floating-Harbor Syndrome: Analysis of Current Original Data and a Review of the Literature. Horm Res Paediatr. 2019;92(2):115-23.

Hood RL, Lines MA, Nikkel SM, Schwartzentruber J, Beaulieu C, Nowaczyk MJ, et al. Mutations in SRCAP, encoding SNF2-related CREBBP activator protein, cause FloatingHarbor syndrome. Am J Hum Genet. 2012; 90(2):308-13.
Hutchinson DT, Sullivan R. Rubinstein-Taybi Syndrome. J Hand Surg Am. 2015;40(8): 1711-2.

Kehrer M, Beckmann A, Wyduba J, Finckh U, Dufke A, Gaiser U, et al. Floating-Harbor syndrome: SRCAP mutations are not restricted to exon 34. Clin Genet. 2014;85(5):498-9.

Ko J, Pomerantz JH, Perry H, Shieh JT, Slavotinek AM, Oberoi S, et al. Case Report of FloatingHarbor Syndrome With Bilateral Cleft Lip. Cleft Palate Craniofac J. 2020;57(1):132-6.

Leisti J, Hollister DW, Rimoin DL. The FloatingHarbor syndrome. Birth Defects Orig Artic Ser. 1975;11(5):305.

Messina G, Atterrato MT, Dimitri P. When chromatin organization floats astray: The Srcap gene and Floating-Harbor syndrome. J Med Genet. 2016;53:793-7.

Nikkel SM, Dauber A, de Munnik S, Connolly M, Hood RL, Caluseriu O, et al. The phenotype of Floating-Harbor syndrome: Clinical characterization of 52 individuals with mutations in exon 34 of SRCAP. Orphanet J Rare Dis. 2013;8:63.
Pelletier G, Feingold M. Case report 1. In: Bergsma D, editor. Syndrome Identification. White Plains: National Foundation-March of Dimes; 1973.p. 8-9.

Seifert W, Meinecke P, Krüger G, Rossier E, Heinritz W, Wüsthof A, et al. Expanded spectrum of exon 33 and 34 mutations in SRCAP and follow-up in patients with Floating-Harbor syndrome. BMC Med Genet. 2014;15:127.

Shields LBE, Peppas DS, Rosenberg E. Renal Calculus in Floating-Harbor Syndrome: A Case Report. J Pediatr Health Care. 2019;33(1):97101.

Vagge A, Desideri LF, Nucci P, Serafino M, Giannaccare $\mathrm{G}$, Lembo A, et al. Congenital Nasolacrimal Duct Obstruction (CNLDO): A Review. Diseases. 2018;22(6):96.

van Genderen MMV, Kinds GF, Riemslag FC, Hennekam RC. Ocular Features in Rubinstein-Taybi Syndrome: Investigation of 24 Patients and Review of the Literature. Br J Ophthalmol. 2000;84:1177-84.

White SM, Morgan A, Da Costa A, Lacombe D, Knight SJ, Houlston R, et al. The phenotype of Floating-Harbor syndrome in 10 patients. Am J Med Genet A. 2010;152A(4):821-9. 